\title{
Scleromalacia perforans as the presenting sign for rheumatoid arthritis - a case report
}

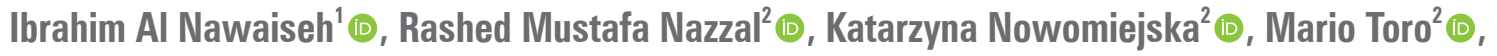 \\ Robert Rejdak ${ }^{2}$ \\ 'Jordan German Eye Centre, Amman, Jordan \\ ${ }^{2}$ Medical University of Lublin, Lublin, Poland
}

\begin{abstract}
Scleromalacia perforans is a rare but well-recognised extra-articular complication of rheumatoid arthritis. It is diagnosed usually late in the course of the disease. In this paper we present a case of scleromalacia perforans in which the patient was not diagnosed with rheumatoid arthritis previously; the elderly female presented with bilateral foreign body sensation and was found to have scleromalacia perforans, which was treated medically with a bolus dose of steroids followed by combined oral steroids and methotrexate, from which she developed the devastating side effect of thrombocytopaenia. The patient was planned for surgical therapy for the scleromalacia, but unfortunately she passed away during the follow-up.
\end{abstract}

KEY WORDS: scleromalacia perforans; rheumatoid arthritis; steroids; hyaline plaque; scleral patch

Ophthalmol J 2019; Vol. 4, 56-59

\section{INTRODUCTION}

Scleritis is an uncommon condition characterised by oedema and cellular infiltration of the entire thickness of the sclera. It is much less common than episcleritis and covers a spectrum ranging in severity from trivial self-limiting episodes to a necrotising disease that may involve adjacent tissues and threaten vision [1]. Scleritis has traditionally been broadly classified into two major categories depending on the cause: infectious and non-infectious. The non-infectious type is mainly immunological and is further subdivided into two main categories: anterior and posterior. The anterior subtype can be either necrotising or non-necrotising, with the former being inflammatory or non-inflammatory [2].

Scleromalacia perforans is a rare $(4 \%$ of scleritis cases) and specific type of anterior necrotising scleritis without inflammation, which typically affects elderly women with longstanding rheumatoid arthritis. The use of the word 'perforans' is unfor- tunate, however, because perforation of the globe is extremely rare as the integrity of the globe is maintained by a thin, but complete, layer of fibrous tissue $[1,2]$.

\section{CASE REPORT}

A 70-year-old female patient, not known to have any chronic medical illness, presented to the clinic complaining of recent bilateral foreign body sensation in both eyes, with gradual blurring of vision over the last few months. Her offspring noted a black spot in her whitish part of the eye developing over the previous few weeks. On examination of the anterior segment of both eyes (Fig. 1) were multiple masses were seen protruding through the sclera representing herniated uveal tissue with very thin conjunctival sheets overlying the masses with diffuse areas of scleral thinning all over the globe. A working diagnosis of anterior necrotising scleritis 


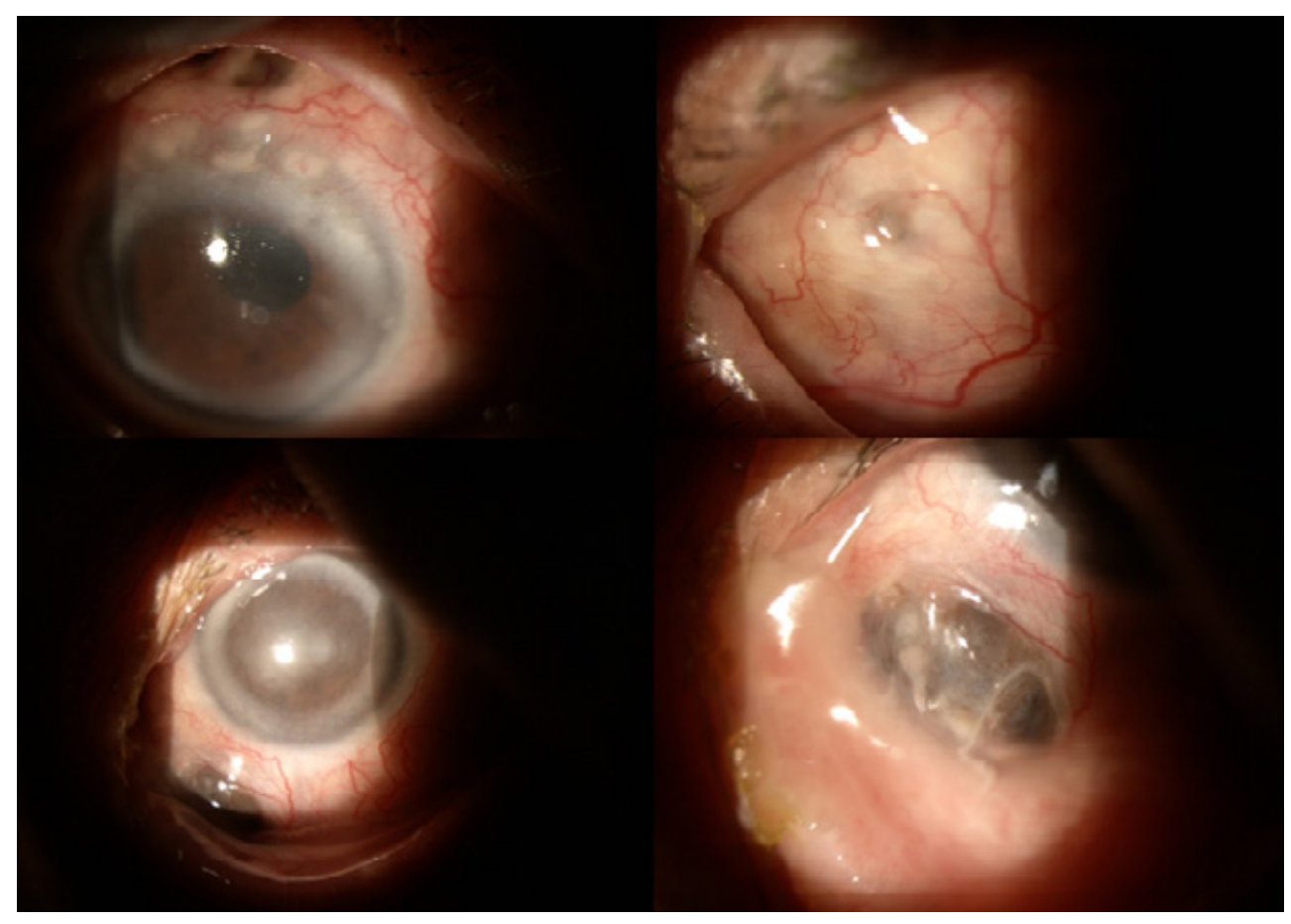

FIGURE 1. Upper row showing the right eye; the lower row showing the left eye

without inflammation (scleromalacia perforans) was made and the patient was started immediately on a bolus dose of steroids (1 $\mathrm{g}$ intravenous methylprednisolone given slowly over 45 minutes with cardiac monitor), and a rheumatological consultation was ordered. The rheumatologist stated that the patient featured a full-blown picture of rheumatoid arthritis (although not previously diagnosed) with agreement for the bolus steroid therapy to be completed in three days. During this time, a wrist X-ray and full serological, immunological, and chemistry tests were ordered. Radiology and blood panel came back consistent with the diagnosis of rheumatoid arthritis. The patient's delayed diagnosis is mainly due to neglect because she was living alone and nobody was taking care of her. The patient was discharged after three days on oral prednisolone tabs $40 \mathrm{mg}$ daily and methotrexate $15 \mathrm{mg}$ once per week (with the addition of folic acid, omeprazole, and vitamin $\mathrm{D}$ supplement). The patient was offered the surgical option (scleral patch over the herniated areas) and was scheduled to undergo surgery within a few days. Unfortunately, she then started to complain of generalised fatigue, and laboratory tests immediately done showed pancytopaenia, which was ascribed to methotrexate therapy. Methotrexate was stopped and the surgery was postponed. The patient was followed up for the following few weeks, wait- ing for her condition to stabilise in order to proceed with surgical treatment for the scleromalacia. Few weeks later, however, she passed away from myocardial infarction.

\section{DISCUSSION}

The description of scleromalacia perforans is very old; Van Der Hoeve in 1931 drew attention to a condition characterised by the spontaneous appearance of scleral perforations, which he named scleromalacia perforans [3]. A few years later he reported similar cases in which he noted that the disease was bilateral in most patients and began in the anterior sclera with yellow or greyish sub-conjunctival nodules, associated with a gradually developing scleral necrosis, progressing to perforation with exposure of the uvea [4]. It is usually difficult to define the onset of the disease because of slow progression and total lack of symptoms. The change in scleral colour is detected by patient's family (as in our case), by the patient looking in the mirror, or by an ophthalmologist during routine examination. Progression of the disease is revealed as necrotic areas without surrounding inflammation, and the bare uvea is covered only by thin layer of conjunctiva $[1,2]$. Although the condition is classically associated with rheumatoid arthritis, it was also described 
in other systemic vasculitis and collagen disorders (up to 66\%) such as systemic lupus erythematosus, periarteritis nodosa, Wegener's granulomatosis, Behçet disease, limited scleroderma, Crohn's disease, graft-versus-host disease, and in porphyria and herpes-zoster infection [5].

Visual loss, secondary to progression of astigmatism (sclera and limbic and corneal changes), anterior uveitis, cataract (secondary to uveitis or steroid therapy), or glaucoma (secondary to ocular abnormalities or steroid therapy) is described generally in the late stages of the scleritis (in up to $60 \%$ of cases) $[1,2]$.

The main differential diagnosis for the disease is the scleral hyaline plaque, which is an oval, dark-greyish area located close to the insertion of the horizontal rectus muscles, which typically affects elderly patients and is innocuous [1].

Regarding the pathophysiology of the disease, autoimmune reaction is responsible for the vessel damage (type III hypersensitivity), which explains why immunosuppressive therapy is suggested to interrupt the destructive process $[1,2,6]$.

Although scleritis may be the initial sign of rheumatoid disease, it usually presents more than 10 years after the onset of arthritis. Multiple studies have found that patients with scleritis have more advanced joint disease and more extra-articular manifestations than do patients with rheumatoid arthritis without scleritis [7]. Although our patient did manifest a full-blown picture of rheumatoid arthritis, she was not diagnosed with the disease earlier, mainly due to neglect.

Treatment may be effective in patients with very early disease, but by the time most patients present, either no treatment is needed or it is unlikely to be effective. Repair of scleral perforation is very difficult but must be attempted otherwise phthisis bulbi ensues. ${ }^{1}$

Although cyclophosphamide is known as the most effective drug in patients with non-infectious necrotising scleritis, other immunosuppressive drugs like methotrexate, azathioprine, cyclosporine, and mycophenolate mofetil are well described [1, 2]. There are some reports describing the following: tumour necrosis factor inhibitors - TNF1 (etanercept, infliximab), the interleukin-2 receptor blocker (daclizumab), the interleukin-1 receptor antagonist (anakinra), and the antilymphocyte medicament (rituximab, alemtuzumab) in managing ocular diseases, including scleritis [8].
To detect and prevent side effects of immunosuppressive therapy, we recommend collaboration with physicians trained in the early recognition and management of drug-induced complication (e.g. haematologist, rheumatologist, internal medicine specialist), as was shown in our case, in which pancytopaenia developed and had to be dealt with by the rheumatologist.

Surgical treatment of scleromalacia is necessary in cases with exposed uvea to preserve the globe integrity. Tectonic patch grafting can be performed with the sclera (fresh or frozen globe or glycerine preserved scleral tissue), dermis, fascia lata, periosteum, aortic tissue, cartilage cornea, pedicle-flaps of conjunctiva with Müller's muscle or tarsus, synthetic material, and eventually amniotic membrane [5].

Patients with scleritis have higher morbidity and mortality rate; if left untreated with systemic medications, up to $45 \%$ of patients with scleritis and rheumatoid arthritis will die within three years of the onset of scleritis. This compares to a three-year mortality rate of $18 \%$ in patients with rheumatoid arthritis but without scleritis. Death is usually secondary to extra-articular vasculitis. Necrotising scleritis is associated with higher mortality than the other forms [9]. Unfortunately, this is what our patient suffered from, resulting in her demise with myocardial infarction.

\section{CONCLUSIONS}

Although it usually presents late in the course of the disease, scleromalacia perforans is sometimes present before the underlying disease is diagnosed or even manifested. Aggressive treatment with immunosuppression therapy is recommended to halt the progression of the disease, and this should be done in liaison with an internist who has a good experience in managing the resultant complications from these agents.

\section{ACKNOWLEDGEMENTS}

None.

\section{STATEMENT OF COMPETING INTERESTS}

No competing interests.

\section{REFERENCES}

1. Jack J, Kanski BB. Clinical Ophthalmology: A Systematic Approach. Elsevier 2016: 253-267.

2. American Academy of Ophthalmology. Basic and Clinical Science Course. Section 8. American Academy of Ophthalmology, San Fransisco 2014: 202-210. 
3. van der Hoeve J, J va. Scleromalacia perforans. Ned T Geneesk. 1931; 75: 4733.

4. Ashton N, Hobbs HE. Effect of cortisone on rheumatoid nodules of the sclera (scleromalacia perforans). Br J Ophthalmol. 1952; 36(7): 373-384, doi: 10.1136/bjo.36.7.373, indexed in Pubmed: 14935113.

5. Kopacz D, Kopacz D. Scleromalacia Perforans - What We Know and What We Can Do. J Clin Exp Ophthal. 2013: 9-11, doi: 10.4172/21559570-s2-009.

6. Fong LP, Sainz de la Maza M, Rice BA, et al. Immunopathology of scleritis. Ophthalmology. 1991; 98(4): 472-479, doi: 10.1016/s01616420(91)32280-2, indexed in Pubmed: 1828871.
7. Ghauri MI, Riaz SU, Husain A, et al. Scleromalacia perforans: a case report. J Med Case Rep. 2018; 12(1): 155, doi: 10.1186/s13256-0181686-z, indexed in Pubmed: 29866188.

8. Herrera-Esparza R, Avalos-Díaz E, Herrera-Esparza R, et al. Infliximab treatment in a case of rheumatoid scleromalacia perforans. Reumatismo. 2009; 61(3): 212-215, indexed in Pubmed: 19888506.

9. Foster CS, Forstot SL, Wilson LA. Mortality rate in rheumatoid arthritis patients developing necrotizing scleritis or peripheral ulcerative keratitis. Effects of systemic immunosuppression. Ophthalmology. 1984; 91(10): 1253-1263, doi: 10.1016/s0161-6420(84)34160-4, indexed in Pubmed: 6514289. 